\title{
Vorwort zur 252. Auflage
}

Enge Zusammenarbeit mit Kliniken aller Fachrichtungen und kritische Auswertung besonders der auf die Klinik und Praxis ausgerichteten Zeitschriften machten auch für diese neue Auflage die Aufnahme einer Reihe neuer Stichwörter notwendig.

Allen mitarbcitenden Autoren spreche ich meinen Dank für ihre Mitwirkung aus. Besonders danke ich Herrn Prof. Dr. A. KELÂmi, Klinikum Steglitz, Berlin, der mich bei der Formulierung wichtiger urologischer Begriffe sehr unterstützte, desgleichen Herrn Dr. H. Modde, La Chaux-de-Fonds, Schweiz, für wertvolle Hinweise auf dem Getiete der Bakteriologie und der Antibiotika, Herrn Dipl.Phys. D. Braun, Berlin, für seine Beratung bei der Neufassung physikalisch-medizinischer Begriffe, Herrn Med. Dir. Dr. G. Raschke, Landesinstitut für Arbeitsmedizin Berlin, für Unterstützung bei der Bearbeitung der Minimatá-Krankheit, der Itai-Itai-Krankheit und der Vinylchlorid-Krankheit. Nicht zuletzt habe ich meiner Frau zu danken, die für die vorliegende Auflage die redaktionelle Arbeit ausführte und mich beim Lesen der Korrekturen unterstützte.

Von interessierten Benutzern des Wörterbuches gingen zahlreiche Zuschriften ein, in denen auf Lücken und verbesserungsbedürftige Texte hingewiesen wurde. Viele dieser kritischen Hinweise und Anregungen waren brauchbar und trugen zur Verbesserung des Buches bei. Bei anderen gingen die Forderungen an den eigentlichen Zielen dieses Wörterbuches vorbei, das in erster Linie den Bedürfnissen der Klinik und der Praxis dienen soll.

Bei einer Anzahl älterer Begriffe wurden notwendige Berichtigungen und Ergänzungen vorgenommen. Überholte Begriffe wurden gestrichen.

Einige Abbildungen sind hinzugekommen, andere wurden verbessert. 


\section{Verzeichnis der Mitarbeiter}

Innere Medizin: Dr. H. HennIng, Chefarzt des Sanatoriums Föhrenkamp der BFA, Mölln (Lauenburg)

Diabetes: Prof. Dr. med. H. Pickert, Ärztlicher Direktor und Chefarzt der II. Inn. Abtlg. des Städt. Krankenhauses Berlin-Spandau

Radiologie: Dr. med. K. Freye, Facharzt für Radiologie, Berlin

Herzchirurgie: Prof. Dr. med. Ferbers, Düsseldorf

Orthopädie: Dr. med. A. PAPE, Facharzt für Orthopädie, Berlin

Dermatologie: Prof. Dr. med. K. Winkler, Oberarzt der Hautklinik des Städt. Krankenhauses, Berlin-Britz

Ophthalmologie: Prof. Dr. med. H. Trвurtius, Oberarzt der Univ.Augenklinik der Freien Universität Berlin

Pädiatrie: Prof. Dr. med. habil. B. Schneeweiss, Chefarzt der Kinderklinik des Städt. Krankenhauses Friedrichshain

1037 Berlin - DDR

Psychiatrie: Dr. med. E. Hirschberg, Facharzt für Psychiatrie und Neurologie, Berlin

Pharmakologie: Dr. med. R. Timmler, Facharzt für Pharmakologic, Mannheim-Waldhof

Psychopharmaka: Prof. Dr. med. K. Kanig, Wiss. Rat, Homburg/ Saar

Endokrinologie: Dr. med. Eva Richter-Bonacker, Berlin

Humangenetik: Prof. Dr. med. F. Vogel, Direktor des Instituts für Anthropologie und Humangenetik der Universität Heidelberg

Biochemie: Dr.med. Joachim Dudenhausen, Berlin (Beratung: Prof. Dr. Dr. Gerhards, Berlin)

Sexualstrafrecht: Staatsanwalt W. FABER, Wattenscheid-Eppendorf Porphyrinurie:Prof. Dr. med. J. Brugsch, Berlin

Zeitschriftenbearbeitung: Dr. med. Elisabeth Praetorius, Kassel

Redaktion: Dr. med. Ingrid Pschyrembel, Oberarzt am Städt. Rudolf-Virchow-Krankenhaus, Berlin 65 
Für Unterstützung, Verbesserungsvorschläge und kritische Hinweise habe ich zu danken den Herren Prof. Dr. med. K. Fr. Bauer, Erlangen; A. BERG, Berliner Medizinische Zentralbibliothek; Dr. BraAtsch, Leverkusen; Prof. Dr. med. Bücklers, M.-Gladbach; D. Degenhardt, Berlin; Prof. Dr. G. Friedebold, Berlin; Prof. Dr. med. Derra, Düsseldorf; Prof. Dr. med. Gasteiger, Berlin; Dr. E. Geyer, Berlin; Frl. Med.Ass. G. Görschrng, Freiburg; Prof. Dr. Dr. E. H. Graul, Marburg; Prof. Dr. K. Gutzeit, Braunschweig; O.-Med.-Rt. Dr. med. Hasse, Berlin; Prof. Dr. med. Hertel, Marburg/L.; Med.Ass. B. Hochhermer, Berlin; Prof. Dr. med. HoffB yuter, Berlin; Med.Ass. G. Hollstein, St. Augustin; Dr. D. JänCr.EN, Muttenz/Schweiz; Prof. Dr. Klose, Wien; Med.Ass. M. MerL, Köln; Dr. med. Scharff, Berlin; Prof. Dr. med. Scheid, Köln; Prof. Dr. med. Schober, München; Prof. Dr. med. Schlungbaum, Berlin; Priv.Doz. Dr. med. G. Schulze-Bergmann, Hamburg-Haarburg; Med.Ass. M. Sires, Beyruth; Oberlehrer N. Stransky, Böheimkirchen; Prof. Dr. med. Vollmar, Ulm; Prof. Dr. med. Volkheimer, Berlin; Prof. Dr. M. Wallach, New York; Oberregierungsrat Weise, Berlin; Med.Ass. G. Wenzel, Freiburg; Dr. Wiechert, Leverkusen; Prof. Dr. med. E. Willich, Heidelberg; Med.-Rt. Dr. med. WinderLICH, Wien; Prof. Dr. med. WrтT, München.

Berlin, Januar 1975

1 Berlin 19, Rüsternallee $45 \quad$ Willibald Pschyrembel 